\title{
UN NUEVO Chthonius CAVERNÍCOLA DE LA PROVINCIA DE ALICANTE (ARACHNIDA: PSEUDOSCORPIONIDEA, CHTHONIIDAE)
}

\author{
por \\ Juan A. Zaragoza ${ }^{1}$
}

\section{ABSTRACT}

A new cave-dwelling specie of Chthonius (Pseudoscorpions, Chthoniidae) from Beniarbeig, Alicante (Spain), is described and figured: Chthonius (E.) mahnerti n. sp.

\section{INTRODUCCION}

La fauna cavernícola de pseudoscorpiones del Levante español se encuentra muy escasamente estudiada, al menos en comparación con otros grupos (coleópteros). Tan solo 5 especies y 1 subespecie han sido descritas como propias de la zona y todas ellas, con una única excepción, se incluyen dentro de la familia Neobisiidae, a la cual asimismo pertenece la primera descripción de una especie levantina: Roncus (P.) boneti, efectuada por BEIER en 1931 y cuyo número se ha visto ampliado considerablemente en tiempos más recientes: MAHNERT 1977, ESTANY 1978 y ZARAGOZA 1982, lo que se ha debido principalmente a una mayor actividad de los recolectores. De la familia Chthoniidae, hasta hoy solo se conocía una especie: Chthonius (E.) ventalloi, BEIER 1939, de la Cueva del Candil en Tous (Valencia) y a la que viene a sumarse la nueva especie aquí descrita. Todo hace pensar que las cavidades subterráneas del Levante deben albergar aún muchas e interesantes novedades.

\section{Chthonius (Ephippiochthonius) mahnerti n.sp.}

Material: Cova del Bolumini, Beniarbeig, Alicante, 27.09.81, legs. Vta. Ripoll \& J. A. Zaragoza: 1 O (Holotipo), 14.12.81, leg. J. Coronado: 1 (Alotipo). (Ambos provisionalmente en la colección del autor).

(1) Colaborador del Departamento de Biologia. Facultad de Ciencias. Universidad de Alicante. 


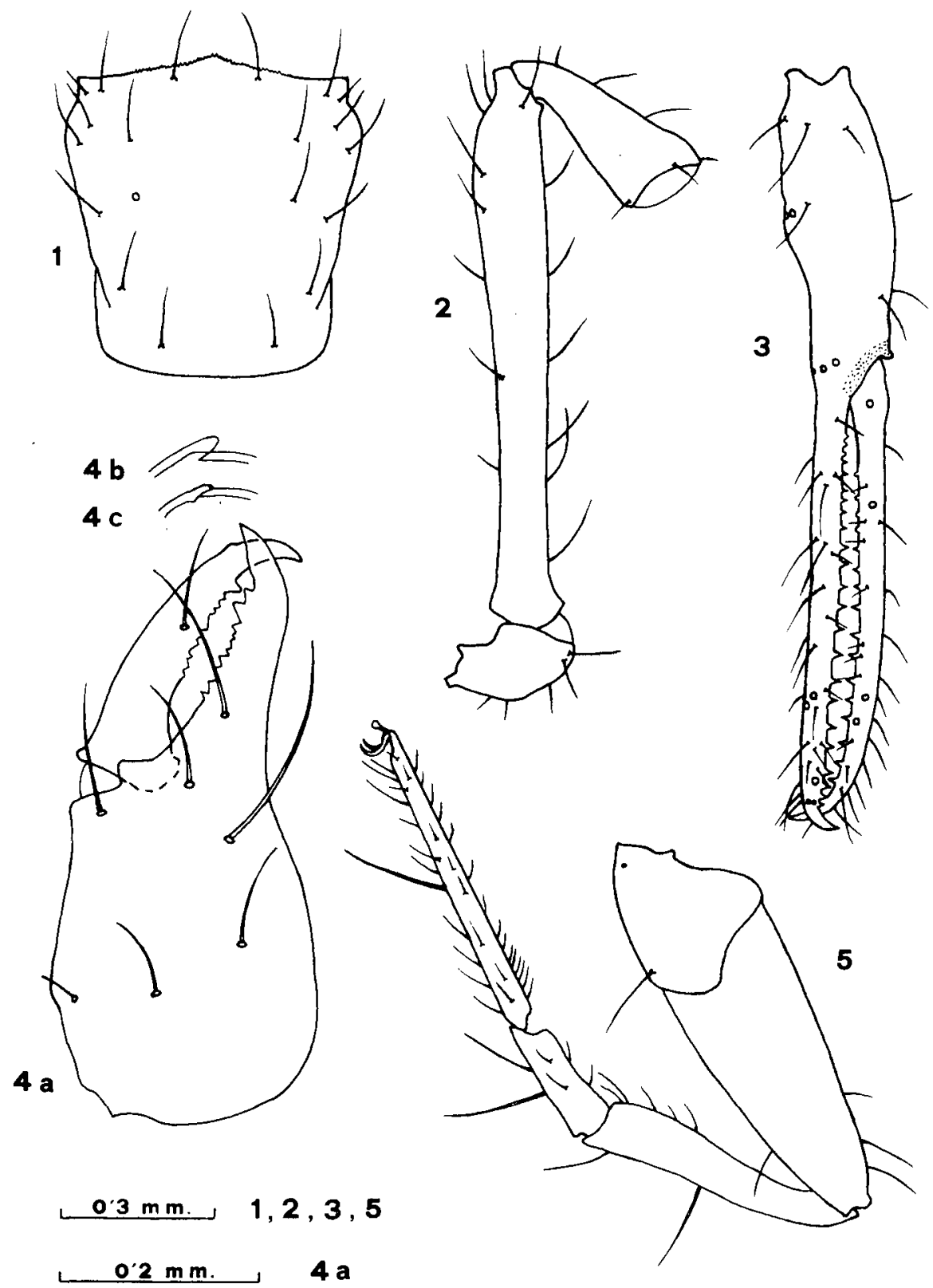

Chthonius (E.) mahnerti n.sp. Holotipo. 1: Carapacho. 2-3: Pedipalpo.

4: a) Quelicero; b) Tubérculo sedicígero de la $\%$; c) Id. del $\sigma^{\circ} .5$ : Pata IV. 
Derivatio nominis. Dedico esta nueva especie al Dr. Volker Mahnert, del Museo de Historia Natural de Ginebra, a quien agradezco profundamente la exquisita amabilidad con que siempre ha atendido mis consultas y me ha ofrecido sus valiosos consejos.

\section{DESCRIPCION}

Carapacho (Figura 1) solo un poco más largo que ancho $\left(0^{*} 1^{\prime} 07 \mathrm{X}\right.$, o 1'10 X), estrechado ligeramente hacia las esquinas posteriores; borde anterior levemente prominente y dentado en el centro; ojos ausentes; con 18 sedas $(4+1$ microseda preocular a cada lado-6-4-2-2) (el Dr. Mahnert me comunica por carta que un ejemplar $q$ que le envié para su revisión, presenta una microseda adicional en la zona posterior, siendo su fórmula quetotáxica: 4 + 1x2-6-4-2-3, tal microseda no aparece sobre los ejemplares aquí descritos); sedas supraoculares tan solo algo más cortas que las demás.

Quetotaxia de los terguitos I-XI: 4-4-4-4-6-6-6-6-6-4-6.

Quelícero (Figura 4a) con 6 sedas en la mano más 1 microseda; dedo fijo con 2 grandes dientes distales y 8 más pequeños $(q: 2+10)$, dedo móvil con 1 gran diente distal y 9 más pequeños $(1+12)$; un diente subapical aislado no está presente; el tubérculo sedicígero aparece en el ơ tan solo como una leve protuberancia (Figura 4c), grande y con forma de diente en la $\$$ (Figura 4b); serrulla externa con 16 láminas, interna con 13 ( $\odot$ : 14); flagelo con 11 sedas unilateralmente aserradas, la más próxima de mitad longitud que las otras.

Lóbulo del pedipalpo con 2 sedas, coxa del pedipalpo con 3, coxa I con $3+3$ microsedas apicales y marginales, II con $4+11-16$ espinas coxales, III con 5+6-8 espinas coxales, IV con 6 , tubérculo intercoxal con 2 sedas.

Opérculo genital en el o* con 9 sedas, abertura genital ancha con $9+8$ sedas en los márgenes, cámara genital con $2 \times 4$ «sedas»; opérculo genital en la $\subsetneq$ con 10 sedas. Quetotaxia de los esternitos III-X : $10+2 \times 3-4$ sedas estigmales ( $\subsetneq: 8+2 \times 3$ ) $-8+2 \times 1-2$ sedas estigmales- 8 (sedas laterales (SL) diminutas)-6 (SL diminutas)-6 (SL diminutas)-6 (SL cortas)-6-7 ( 2 sedas tactiles submediales). 
Pedipalpos (Figs. 2-3): trócanter $1^{6} 74 \mathrm{X}$ más largo que ancho en el $\mathrm{O}^{\prime}$ ( $ᄋ$ : $1 ‘ 81 \mathrm{X})$; fémur $8 \mathrm{X}\left(8^{`} 23 \mathrm{X}\right), 2^{`} 60 \mathrm{X}\left(2^{`} 57 \mathrm{X}\right)$ más largo que la tibia y 1‘75 X más largo que el carapacho; tibia 2‘50 X (2‘44 X); mano 2‘80 X $(2 ‘ 75 \mathrm{X})$, distal de $i b / i s b$ con depresión amplia y poco profunda; dedo 1‘64 X más largo que la mano (1‘55 X); pinzas 7‘39 X (7‘00 X). Tricobotrio ist casi a la misma altura de esb. Dedo fijo con 20 (21) dientes puntiagudos situados separadamente, el más distal pequeño y los 13 (15) inmediatamente siguientes grandes, aproximadamente de doble tamaño que los otros 6 (5), después de éstos aún 3 pequeños dientes redondeados más en posición basal; apicalmente un diente en posición lateral. Dedo móvil con 12 (13) dientes puntiagudos, los 9 primeros distales grandes y los 3 (4) siguientes más pequeños, todos bien separadamente situados y distales de $e s b$, a continuación y hacia la base 4 (3) pequeños dientes redondeados más.

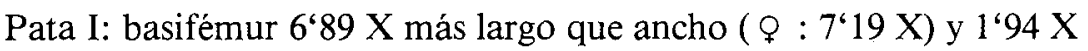
$\left(1^{‘} 93 \mathrm{X}\right)$ más largo que el telofémur, éste $4 \mathrm{X}\left(3^{‘} 96 \mathrm{X}\right)$; tibia 5‘20 X $\left(5^{`} 50 \mathrm{X}\right)$; tarso $11^{`} 21 \mathrm{X}\left(12^{`} 31 \mathrm{X}\right)$ y $2^{`} 02 \mathrm{X}\left(1^{`} 94 \mathrm{X}\right)$ más largo que la tibia.

Pata IV (Figura 5): fémur 3'22 X ( $9: 3 \mathrm{X})$, tibia 5‘32 X (4’96 X);

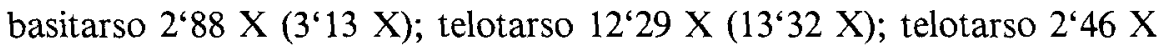
$\left(2^{\circ} 41 \mathrm{X}\right)$ más largo que el basitarso. Seda tactíl (ST) del basitarso cercana a la mitad: $0^{`} 45\left(0^{\star} 46\right)$, ST del telotarso en la mitad o cercana a ella: $0^{`} 49$ $\left(0^{`} 42\right)$.

Medidas del cuerpo: en mm.

Longitud del cuerpo: ơ 2‘04 ( $\%: 2^{‘} 06$ ).

Carapacho: 0‘64-0‘59 (0‘64-0‘58).

Pedipalpos: fémur 1‘12-0`14, tibia 0`43-0`17 (0`43-0`18), mano 0`58-0`21 (0`60-0`22), dedo 0`95 (0`93).

Pata I: basifémur 0`61-0`09 (0‘58-0`08), telofémur $00^{`} 31-0^{`} 08\left(0^{`} 30-0 ` 08\right)$, tibia $0^{\circ} 32-0^{`} 06\left(0^{`} 33-0^{`} 06\right)$, tarso $0^{`} 64-0^{`} 06\left(0^{`} 63-0^{`} 05\right)$.

Pata IV: fémur 0`87-0‘27 (0`86-0`29), tibia 0`57-0‘11 (0‘59-0`12), basitarso $0^{\circ} 27-0^{\circ} 09\left(0^{\circ} 28-0^{\circ} 09\right)$, telotarso 0`65-0‘05 (0`66-0`05). 
Especie que parece bien adaptada al medio ambiente subterráneo, por lo que puede considerársela un auténtico troglobio.

\section{DISCUSION}

La nueva especie Chthonius (E.) mahnerti presenta afinidades con otras especies cavernícolas de la Península Ibérica y alguna otra del Mediterráneo Noroccidental, particularmente con grafittii GARDINI (isla de Cerdeña), de la que se diferencia, entre otros detalles, por la dentición de los dedos de la pinza, la quetotaxia del carapacho, la forma del tubérculo sedicígero del or $^{\prime}$ y la posición de la seda tactíl en el telotarso de la pata IV. De entre las especies ibéricas, se aleja claramente de ventalloi BEIER (la especie geográficamente más próxima) por la ausencia en mahnerti de lámina basal en el dedo móvil de la pinza y el relieve dorsal de la mano; de hiberus BEIER y nudipes MAHNERT se distingue bien por las proporciones de los pedipalpos. Más cercano a balearicus MAHNERT (isla de Mallorca) del que se separa por: la ausencia total de ojos en mahnerti, borde anterior del carapacho leve pero claramente prominente (completamente recto en balearicus), la ausencia de tubérculo sedicígero en el $\sigma^{\prime}$ de la especie balear, la mano y la pinza más esbeltas en la nueva especie, diferente dentición de los dedos de la pinza, la seda tactíl del telotarso de la pata IV en la mitad o muy cerca de ella (en el tercio basal en balearicus) y la ausencia en mahnerti de microsedas accesorias sobre la línea ocular.

\section{BIBLIOGRAFIA}

BEIER, M. 1930. Neue Höhlen-Pseudoscorpione der Gattung Chthonius. Eos, Madrid, tomo VI: $323-327$.

BEIER, M. 1931. Zur Kenntnis der troglobionten Neobisien (Pseudoscorp.). Eos, Madrid, tomo VII, 1:: 9-23.

BEIER, M. 1939. Die Pseudoscorpioniden-Fauna der iberischen Halbinsel. Zoologische Jahrbücher (Systematik), Jena 72 (3/4): 157-202.

BEIER, M. 1963. Ordnung Pseudoscorpionidea (Afterskorpione). Bestimmungsbücher zur Bodenfauna Europas, Berlin, 313 pp.

ESTANY, J. 1978. Sobre algunos Neobisiidae cavernícolas del País Valenciano. Speleon, Barcelona, vol. 24: 33-37. 
GARDINI, G. 1981. Pseudoscorpioni cavernicoli sardi. I. Chthoniidae. Revue Arachnologique, París, 3 (3): 101-114.

MAHNERT, V. 1977. Spanische Höhlenpseudoskorpione. Miscelánea Zoològica, Barcelona, vol. IV, fasc. 1: 61-104.

MAHNERT, V. 1982. Neue höhlenbewohnende Pseudoskorpione aus Spanien, Malta und Griechenland (Arachnida, Pseudoscorpiones), Mitteilungen der Schweizerischen Entomologischen Gesellschaft, Zürich, 55: 297-304.

ZARAGOZA, J. 1982. Roncus (Parablothrus) setosus n.sp., otro caso de «néochétotaxie majorante prosomatique» HEURTAULT en los pseudoscorpiones Neobisiidae. Mediterránea, Alicante, 6: 101-108. 